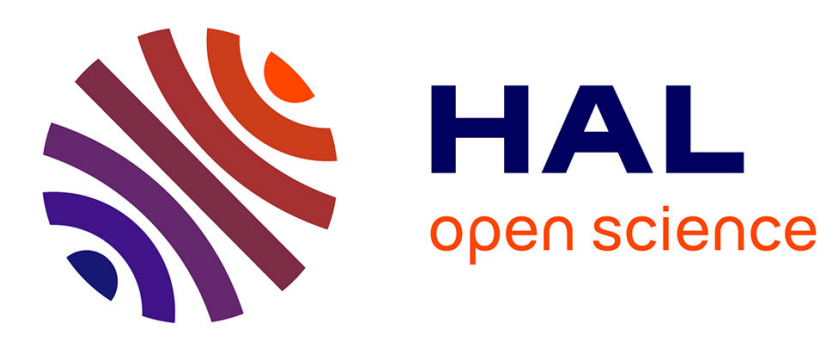

\title{
Calcul du rayonnement acoustique de structures par décomposition sur des fonctions radiatives
}

\author{
J.-J. Laissus, Jean-Louis Guyader
}

\section{To cite this version:}

J.-J. Laissus, Jean-Louis Guyader. Calcul du rayonnement acoustique de structures par décomposition sur des fonctions radiatives. Journal de Physique IV Proceedings, 1994, 04 (C5), pp.C5-85-C5-88. 10.1051/jp4:1994511 . jpa-00252784

\section{HAL Id: jpa-00252784 https://hal.science/jpa-00252784}

Submitted on 1 Jan 1994

HAL is a multi-disciplinary open access archive for the deposit and dissemination of scientific research documents, whether they are published or not. The documents may come from teaching and research institutions in France or abroad, or from public or private research centers.
L'archive ouverte pluridisciplinaire HAL, est destinée au dépôt et à la diffusion de documents scientifiques de niveau recherche, publiés ou non, émanant des établissements d'enseignement et de recherche français ou étrangers, des laboratoires publics ou privés. 


\title{
Calcul du rayonnement acoustique de structures par décomposition sur des fonctions radiatives
}

\author{
J.-J. LAISSUS et J.-L. GUYADER
}

Laboratoire Vibrations et Acoustique (L.V.A.), INSA de Lyon, 20 avenue Albert Einstein, 69621 Villeurbanne cedex, France

\begin{abstract}
In this paper, a superposition method is presented that allows the computation of acoustic fields radiated by vibrating structures . Basically, the method of superposition applied to sound radiation consists in modelizing the vibrating structure by fictitious acoustic sources located inside it. The sources used can be punctual ones such as monopoles or dipoles ; we have developped surfaciq sources which have the advantage of a large spatial bandpass .By a numerical procedure, complex amplitudes of these sources are determined so as to satisfy the boundary conditions, usually normal velocity which is either calculated or measured .Usually authors try to minimize the difference between the known velocity and the approximated one in the least mean square sense. This method implies numerical peculiarities since it leads to ill-conditionned linear equations system. The convergence criterium in terms of velocity is very hard to achieve and it does not take into account radiation efficiency. We present a method in which we add the acoustic strength of the structure as an auxiliary condition.
\end{abstract}

\section{INTRODUCTION}

Dans ce papier nous présentons une méthode de superposition appliquée au calcul du rayonnement acoustique d'une structure dont on connait les vitesses normales sur la frontière. Le champ de pression inconnu est développé sur une base de fonctions radiatives construites à partir de solutions fondamentales de l'équation de Helmholtz .Les amplitudes complexes de ces sources sont déterminées via un procédé de minimisation de l'écart quadratique entre les vitesses imposées et les vitesses reconstruites sur la frontière. Le critère de convergence généralement adopté par les auteurs ( ref.1 à 4 ) est l'erreur quadratique en vitesse. Or ce critère est très pénalisant numériquement et dans le cas d'une convergence imparfaite risque de ne pas tenir compte d'informations traduisant l'efficacité de rayonnement de la structure .Nous introduisons une condition supplémentaire qui impose la conservation du débit acoustique de la structure dans la solution approchée. Cette condition nous conduit à un procédé de minimisation sous contrainte. Nous présentons une application à une plaque non bafflée excitée ponctuellement .Les vitesses vibratoires ont été mesurées et constituent les données de frontière entrées dans le modèle.

\section{DEVELOPPEMENTS THEORIQUES}

\subsection{Problème de rayonnement}

Nous considérons un objet de forme quelconque, dont la normale $\mathbf{n}_{\mathbf{s}}$ et le déplacement normal $\mathbf{w}_{\mathbf{n}}$ sont spécifiés en tout point de sa frontière $S$. Le problème de rayonnement en espace infini est formulé selon le système d'équations 1 à 3 .

$\Delta \mathbf{p}(\mathbf{M})+\mathbf{k}^{2} \mathbf{p}(\mathbf{M})=0$ 
Conditions de Sommerfeld

$\frac{\partial \mathbf{p}\left(\mathbf{M}_{\mathbf{s}}\right)}{\partial \mathbf{n}_{\mathbf{s}}}=\rho \omega^{2} \mathbf{w}_{\mathbf{n}}$

\subsection{Résolution par décomposition sur une base de fonctions radiatives}

L'objet rayonnant est remplacé par un ensemble de $N$ sources radiatives disposées à l'intérieur du domaine fermé délimité par la frontière $S$. Dans le cas où la frontière $S$ n'est pas fermée (c'est le cas d'une plaque non bafflée que nous traiterons dans ce papier) les sources radiatives sont localisées dans l'épaisseur de la structure. Ces sources sont construites à partir de solutions fondamentales de l'équation de Helmholtz. De par ce choix les équations (1) et (2) sont résolues .

La pression en un point $M$ du domaine extérieur à $S$ et le déplacement en un point Ms de la surface $S$ s'expriment selon les équations (4) et (5).

$\mathbf{p}(\mathbf{M})=\sum_{\mathrm{j}=1}^{\mathrm{N}} \mu_{\mathrm{j}} \cdot \psi_{\mathrm{j}}(\mathbf{M})$

$$
\widetilde{\mathbf{w}_{\mathbf{n}}}\left(\mathbf{M}_{\mathbf{s}}\right)=\frac{1}{\rho \omega^{2}} \sum_{\mathbf{j}=1}^{\mathbf{N}} \mu_{\mathrm{j}} \cdot \frac{\partial \psi_{\mathrm{j}}\left(\mathbf{M}_{\mathbf{s}}\right)}{\partial \mathbf{n}_{\mathbf{s}}}
$$

\section{Elaboration des sources radiatives :}

Les sources radiatives sont construites à partir de solutions fondamentales de (1). Elles peuvent être ponctuelles (dipôles, monopôles ) ou étendues. Ce dernier type de source est construit à partir de distributions surfaciques de dipôles ou monopôles pondérés en amplitude par une fonction gaussienne, sur des surfaces élémentaires $S_{j}$.

\section{Minimisation de l'écart quadratique en déplacements :}

Dans ce cas les coefficients complexes $\mu_{\mathbf{j}}$ sont déterminés par résolution de l'équation 3 au sens des moindres carrés , ce qui conduit à rendre minimum la fonctionnelle d'écart $\Phi$ par rapport à toutes les variables $\mu_{\mathbf{j}}$.

$$
\Phi\left(\mu_{\mathbf{j}}\right)=\iint_{\mathbf{S}}\left|\mathbf{w}_{\mathbf{n}}\left(\mathbf{M}_{\mathbf{S}}\right)-\widetilde{w_{n}}\left(M_{S}\right)\right|^{2} d S
$$

Nous sommes amenés à résoudre un système d'équations linéaires d'inconnues $\mu_{\mathbf{j}}$.

$$
\begin{aligned}
& {\left[\alpha_{j k}\right]\left[\mu_{k}\right]=\left[\beta_{j}\right]} \\
& \alpha_{\mathbf{j k}}=\iint_{\mathrm{S}} \frac{\partial \psi_{\mathrm{j}}\left(\mathbf{M}_{\mathbf{S}}\right)}{\partial \mathbf{n}_{\mathrm{S}}} \cdot \frac{\partial \psi_{\mathrm{k}}{ }^{*}\left(\mathbf{M}_{\mathrm{S}}\right)}{\partial \mathbf{n}_{\mathrm{S}}}, \mathrm{dS} \quad \beta_{\mathrm{j}}=\iint_{\mathrm{S}} \rho \omega^{2} \mathbf{w}_{\mathbf{n}}\left(\mathbf{M}_{\mathbf{S}}\right)^{*} \frac{\partial \psi_{\mathrm{j}}\left(\mathbf{M}_{\mathbf{S}}\right)}{\partial \mathbf{n}_{\mathrm{S}}} \cdot \mathbf{d S}
\end{aligned}
$$

Minimisation de l'écart quadratique en déplacements sous contrainte de débit :

Une condition auxiliaire doit être résolue avec l'équation 3 . Cette condition traduit la conservation du débit acoustique de la structure, elle s'écrit selon l'équation (8) .

$$
\iint_{S}\left(\widetilde{w_{n}}\left(M_{S}\right)-w_{n}\left(M_{S}\right)\right) \cdot d S=0
$$

La substitution de (5) dans (8) permet d'exprimer un degré de liberté $\mu_{\mathrm{q}}$ en fonctions des autres $\mu_{\mathrm{j}}$.

$$
\mu_{\mathrm{q}}=\mathbf{f}\left(\mu_{1}, \mu_{2}, \ldots, \mu_{\mathrm{j}}\right) \quad \mathbf{j} \neq \mathbf{q}(9)
$$

Nous obtenons un système d'équations linéaires de taille $\mathrm{N}-1$,qui fait intervenir des fonctions radiatives $\psi_{j}$ ' modifiées et des déplacements $w_{n}$ ' modifiés . 


$$
\begin{aligned}
& {\left[\alpha_{\mathbf{j k}}\right]\left[\mu_{\mathbf{k}}{ }^{\prime}\right]=\left[\beta_{\mathbf{j}}\right]} \\
& \alpha_{\mathbf{j k}}=\iint_{s} \frac{\partial \psi_{\mathbf{j}}\left(\mathbf{M}_{\mathbf{S}}\right)}{\partial \mathbf{n}_{\mathbf{S}}} \cdot \frac{\partial \psi_{\mathbf{k}}{ }^{*}\left(\mathbf{M}_{\mathrm{S}}\right)}{\partial \mathbf{n}_{\mathrm{S}}} \cdot \mathrm{dS} \quad \beta_{\mathbf{j}}=\iint_{S} \rho \omega^{2} \mathbf{w}_{\mathbf{n}}\left(\mathbf{M}_{\mathbf{S}}\right)^{*} \frac{\partial \psi_{\mathbf{j}}\left(\mathbf{M}_{\mathbf{S}}\right)}{\partial \mathbf{n}_{\mathrm{S}}} \cdot \mathrm{dS}
\end{aligned}
$$

Cette méthode a l'inconvénient de briser la symétrie de la base de fonctions que nous utilisons pour décomposer le champ de pression acoustique. Ceci peut inđuire une déformation du diagramme de directivité . Si la contrainte de débit est bien respectée ( ceci dépend de la finesse de discrétisation de la surface et de la précision des phases des vitesses ) la puissance rayonnée sera conservée pour des fréquences situées en dessous de la fréquence critique.Un problème apparait cependant quant au choix arbitraire de la source d'indice q que l'on rend dépendante des autres, on constate que le champ de pression calculé dépend de ce choix.

\section{Minimisation de l'écart quadratique en déplacements sous contrainte de débit à l'aide de multiplicateurs de Lagrange :}

Un moyen d'éviter le problème évoqué précédemment est l'utilisation de la technique des multiplicateurs de Lagrange . Nous construisons une fonctionnelle d'écart modifiée $\Phi$ 'égale à la somme de la fonctionnelle initiale $\Phi$ plus l'équation de débit (8) pondérée par un multiplicateur indéterminé $\lambda$. Nous sommes ainsi ramenés à un calcul variationnel libre qui consistera à minimiser la fonctionnelle modifiée $\Phi^{\circ}$ par rapport aux variables $\mu_{j}$ et $\lambda$. Une inconnue supplémentaire $\lambda$ est introduite, le système est de taille $\mathrm{N}+1$.

$\Phi\left(\mu_{j}, \lambda\right)=\iint_{s}\left|w_{n}\left(M_{S}\right)-\widetilde{w_{n}}\left(M_{S}\right)\right|^{2} \mathbf{d S}+\operatorname{Re}\left\{\lambda \cdot \iint_{\mathbf{s}}\left(\widetilde{w_{n}}\left(M_{S}\right)-w_{n}\left(M_{S}\right)\right) \cdot d S\right\}$

\section{SIMULATIONS NUMERIQUES - CONCLUSION}

Cette méthode a été appliquée au cas d'une plaque non bafflée (fig.1), excitée ponctuellement sur laquelle nous avons mesuré les vitesses en 90 points.Afin de modéliser une structure non bafflée et non déformable dans l'épaisseur des sources de type dipolaire sont utilisées.

La figure 2 présente les dérivées normales des fonctions radiatives pour les deux types de sources , ponctuelles et pistons gaussiens. Dans le cas d'une condition de vitesse sur la frontière $S$ les dérivées normales des fonctions de base vont jouer un rôle de filtre spatial sur les informations vibratoires Comme les sources sont utilisées dans leur champ proche ,leur bande passante est très étroite dans le cas de sources ponctuelles ; un nombre important sera nécesaire pour résoudre notre problème .Il est donc intéressant d'utiliser des sources surfaciques qui permettent de réduire la taille des systèmes à résoudre et le nombre d'informations vibratoires.

L'application de la contrainte de débit selon les équations (9) et (10) montre l'influence de la prise en compte de cette condition sur le champ de pression proche à $2 \mathrm{~cm}$ de la plaque (fig.3 et 4) .Le niveau de pression est très augmenté et la répartition spatiale est très perturbée. Quelques simulations avec l'équation (11) ont montré la supériorité de la technique des multiplicateurs pour reproduire une bonne distribution spatiale du champ de pression. Plusieurs comparaisons avec les résultats expérimentaux en termes de pression proche, puissance et pression en champ lointain seront présentées lors de l'exposé .

Nous remercions les organismes qui soutiennent cette étude, Electricité de France Etudes et Recherches (Paris) ,Renault Division Recherche (Paris), Ministère de la Recherche et de I'Espace.

\section{Références:}

1 L.Cremer und M.Wang ,Acustica vol.65, N²,pp 53-74

2 M.Ochman ,Acustica, vol.72(1990),pp 233-246

3 G.H.Koopman,L.Song,J.B.Fahnline,JASA 86(6), Dec. 1989 ,pp 2433-2438

4 J.J.Laissus,J.L.Guyader Proc. Inter.Noise 93 vol III,pp 1877-1880

5 T.Tomilina Proc. Inter.Noise 93 vol III, pp 1597-1600 


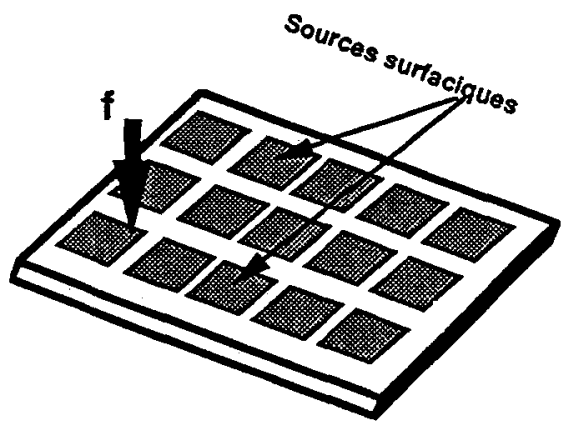

fig. 1 : plaque non bafflée

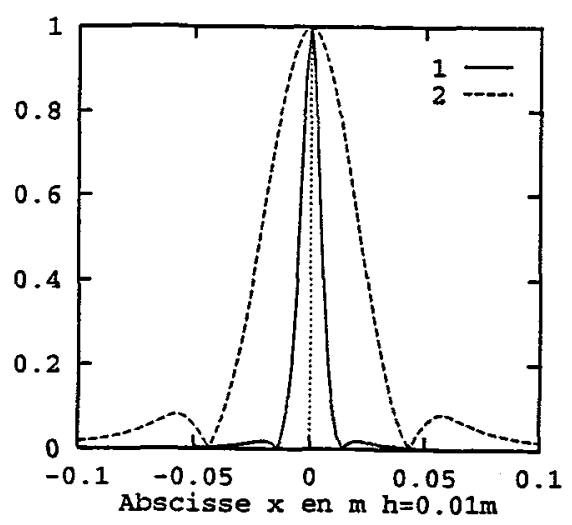

fig. 2: Dérivée normale des fonctions radiatives 1 : dipole 2 : piston gaussien

Modele 49 sources surfaciques $f=1258 \mathrm{~Hz}$

Lp dB ref. 2.0e-05 Pa

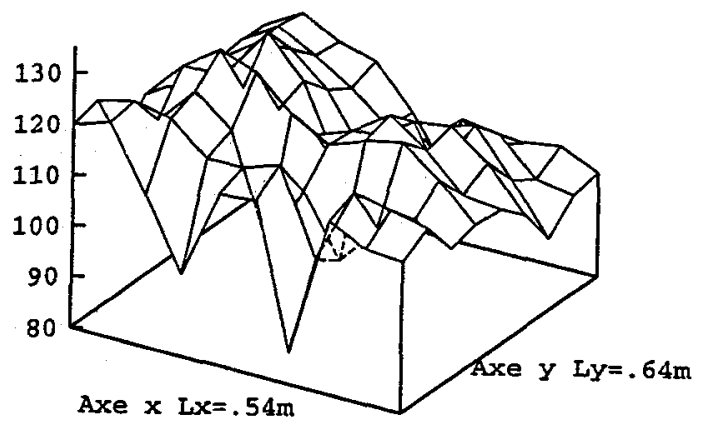

fig. 3: Pression proche $2 \mathrm{~cm}$ calcul sans contrainte de débit

Lp dB ref. 2.0e-05 Pat

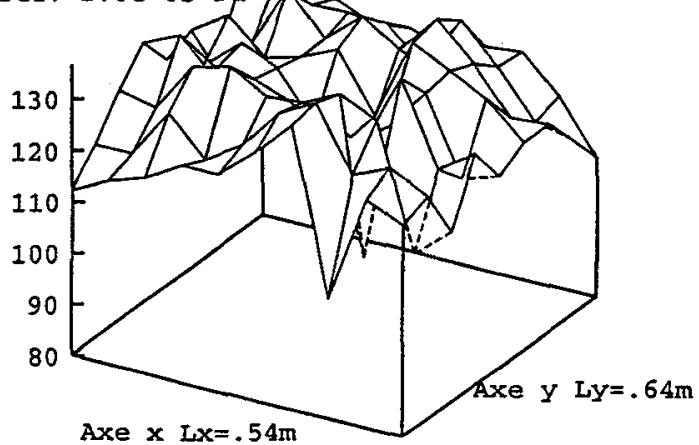

fig. 4 : Pression proche a $2 \mathrm{~cm}$ calcul avec contrainte de débit 